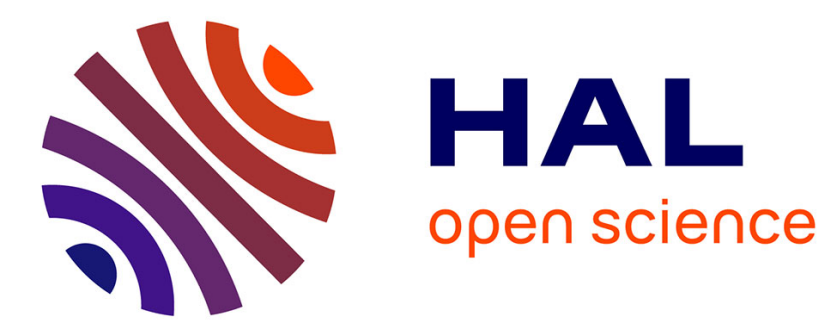

\title{
Origines et développement de la robotique
}

\author{
Nathalie Lazaric
}

\section{To cite this version:}

Nathalie Lazaric. Origines et développement de la robotique. Revue d'économie industrielle , 1992, 61, pp.54-67. hal-00457144

\section{HAL Id: hal-00457144 https://hal.science/hal-00457144}

Submitted on 18 Feb 2010

HAL is a multi-disciplinary open access archive for the deposit and dissemination of scientific research documents, whether they are published or not. The documents may come from teaching and research institutions in France or abroad, or from public or private research centers.
L'archive ouverte pluridisciplinaire HAL, est destinée au dépôt et à la diffusion de documents scientifiques de niveau recherche, publiés ou non, émanant des établissements d'enseignement et de recherche français ou étrangers, des laboratoires publics ou privés. 


\title{
Persée
}

\author{
http://www.persee.fr
}

\section{Origines et développement de la robotique}

\author{
Nathalie LAZARIC \\ Revue d'économie industrielle, Année 1992, Volume 61, Numéro 1 \\ p. $54-67$
}

Voir l'article en ligne

\section{Avertissement}

L'éditeur du site " PERSEE » - le Ministère de la jeunesse, de l'éducation nationale et de la recherche, Direction de l'enseignement supérieur, Sous-direction des bibliothèques et de la documentation - détient la propriété intellectuelle et les droits d'exploitation. A ce titre il est titulaire des droits d'auteur et du droit sui generis du producteur de bases de données sur ce site conformément à la loi n`98-536 du 1 er juillet 1998 relative aux bases de données.

Les oeuvres reproduites sur le site «PERSEE » sont protégées par les dispositions générales du Code de la propriété intellectuelle.

Droits et devoirs des utilisateurs

Pour un usage strictement privé, la simple reproduction du contenu de ce site est libre.

Pour un usage scientifique ou pédagogique, à des fins de recherches, d'enseignement ou de communication excluant toute exploitation commerciale, la reproduction et la communication au public du contenu de ce site sont autorisées, sous réserve que celles-ci servent d'illustration, ne soient pas substantielles et ne soient pas expressément limitées (plans ou photographies). La mention Le Ministère de la jeunesse, de l'éducation nationale et de la recherche, Direction de l'enseignement supérieur, Sous-direction des bibliothèques et de la documentation sur chaque reproduction tirée du site est obligatoire ainsi que le nom de la revue et- lorsqu'ils sont indiqués - le nom de l'auteur et la référence du document reproduit.

Toute autre reproduction ou communication au public, intégrale ou substantielle du contenu de ce site, par quelque procédé que ce soit, de l'éditeur original de l'oeuvre, de l'auteur et de ses ayants droit.

La reproduction et l'exploitation des photographies et des plans, y compris à des fins commerciales, doivent être autorisés par l'éditeur du site, Le Ministère de la jeunesse, de l'éducation nationale et de la recherche, Direction de l'enseignement supérieur, Sous-direction des bibliothèques et de la documentation (voir http://www.sup.adc.education.fr/bib/ ). La source et les crédits devront toujours être mentionnés. 


\section{ORIGINES ET DÉVELOPPEMENT DE LA ROBOTIQUE}

"Le réexamen de notre histoire technologique et économique est un moyen efficace de s'attaquer à la fois aux sources d'inquiétude qui sont sans fondement et à celles qui sont légitimes, et d'arriver par là à une compréhension plus profonde des processus et des conséquences liés à la diffusion des innovations industrielles "évinçant" la main d'œuvre. Je ne suggère pas que, sur ce point, "l'histoire se répète" plus que sur d'autres, mais simplement qu'une mise en perspective historique peut être d'une aide appréciable pour corriger les distorsions introduites par l'inévitable étroitesse d'un point de vue dominé par le présent. »

P.A. David, « La moissonneuse et le robot », Economica, 1986.

\section{INTRODUCTION $(*)$}

L'origine technologique des robots ne remonte pas uniquement aux créations de la firme Unimation, mais aux différentes inventions bien antérieures. Pour retracer cette genèse nous nous appuierons notamment sur le modèle de B. Arthur, montrant les formes d'irréversibilité que peuvent prendre certains développements technologiques à partir de petits évènements provoquant des mécanismes d'autorenforcement. Nous soulignerons d'autre part que si le cadre " arthurien " s'avère utile pour retracer l'histoire des trajectoires technologiques, il peut être néanmoins élargi en introduisant les acteurs de ce processus, les institutions et formes particulières d'organisation inter-firmes qui participent à la construction d'irréversibilité, tout en étant impliquées dans la conception et la sélection technologique. Ainsi cette création - selon l'acception de M. Amendola et J.-L. Gaffard - est issue d'un développement complexe où certains acteurs ont un rôle privilégié, les firmes automobile qui dans de nombreux cas guident l'innovation à partir de leur propre besoin.

Avant d'aller plus loin dans ce débat nous proposons d'examiner plus attentivement dans un premier temps les premières créations qui participèrent au développement de la robotique, dans un second temps l'électronisation du robot industriel pour voir enfin le rôle paradoxal de l'émergence de nouvelles technologies issues de l'électronique dans les principales firmes utilisatrices. 


\section{I. - DES AUTOMATES LUDIQUES À L'ÉMERGENCE DE LA ROBOTIQUE}

Les premières solutions techniques des robots se trouvent dans les mécanismes des automates qui comprenaient déjà quelques principes d'autorégulation. Cependant la différence réside dans la programmation : les robots sont reprogrammables et multifonctionnels selon la tâche impartie, l'automate par contre est simplement animé d'un mécanisme intérieur assurant son mouvement et limitant sa programmation. Leur finalité d'autre part est radicalement opposée, le robot est une machine industrielle tandis que la construction de l'automate repose sur une élaboration ludique, voire philosophique : la recherche du mouvement perpétuel.

\section{Les premiers automates}

La naissance du robot industriel est ancienne et découle de l'ère des objets non finalisés créés par les ingénieurs grecs et arabes. Les Grecs et les Alexandrins tout en réutilisant les principes techniques mis au point par les Égyptiens, compliquent ces mécanismes en apportant des solutions techniques originales (1). Héron d'Alexandrie notamment lors de la mise au point de sa fontaine à vin conçoit le système de régulation d'un liquide qui permet de mieux maîtriser les fluides (2).

Si ces ingénieurs constituent un nouveau corps de savoir autour de la mécanique grâce à la mise au point d'outils tels que la vis et le treuil et leur principe de transmission, ils ne disposent pas, comme le souligne B. Gille d'un " dispositif matériel »- selon l'acception d'E. Zuscovitch - suffisant pour utiliser les matériaux appropriés tels que la tôle ou l'acier et ne peuvent pas changer d'échelle de production (3).

Plusieurs siècles plus tard, les Arabes perpétuent les savoir-faire hérités des Grecs et des Alexandrins. Al Jazari dans son traité d'automates développe certaines solutions existantes au niveau des fontaines tout en se focalisant sur des créations originales telles que les systèmes de transmission et de puissance. Il met notamment au point le système de bielle manivelle qui est la première tentative de transformer un mouvement continu en un mouvement alternatif. Ainsi, si de l'antiquité égyptienne à la mécanique arabe il existe une certaine continuité des principes techni-

(1) Pour plus de précisions à ce sujet voir B. Jacomy (1990), On trouve dans cette approche un panorama assez complet de la vie et de l'œuvre d'Héron d'Alexandrie (deuxième siècle avant notre ère), d'Al Jazari (douzième siècle) et de Vaucanson (1709-1782).

Voir aussi B. Gille, « Les mécaniciens grecs ", Éd. du Seuil, 1980.

(2) Le principe de régulation autonome est la définition que l'on donne à tout automatisme, c'est la base et l'" ancêtre " du système de commande du robot industriel qui comprend une autorégulation ou rétro-action indépendante.

(3) Pour E. Zuscovitch (1984) une solution technique ou innovation est composée de trois éléments : la fonction c'est-à-dire le besoin technique (un problème technique est dû à un besoin économique), le corps de savoir qui est l'aptitude de résoudre des problèmes techniques selon des procédures spécifiques et le dispositif matériel qui permet au corps de savoir de répondre aux besoins techniques en faisant intervenir différentes spécialités industrielles. 
ques, la Renaissance constitue une nouvelle époque car ses innovations ne s'inspirent que très partiellement de cet héritage (4). Cette période s'appuie plus sur la lente structuration des connaissances scientifiques dans la mécanique et les mathématiques et sur la genèse d'un nouveau corps de savoir : l'horlogerie. L'amélioration du dispositif matériel permet notamment à Léonard de Vinci de concevoir de nombreux automates (5).

La Renaissance est une période charnière entre une séquence d'essais technologiques non finalisés (avec la floraison d'automates ludiques) et une séquence où la finalité des objets est plus spécifique et où il y a une structuration plus prononcée des connaissances (les $\mathrm{XVII}^{\mathrm{e}}$ et $\mathrm{XVIII}{ }^{\mathrm{e}}$ siècles sont notamment parsemés de questionnements sur la vitesse, les forces et la résistance des matériaux) (6). Les créations de Vaucanson sont l'aboutissement du XVIII ${ }^{\mathrm{e}}$ siècle et représentent le perfectionnement de solutions techniques déjà éprouvées à travers la mise au point d'automates, parallèlement à la réalisation d'automatismes industriels simples pour réorganiser l'industrie de la soie [B. Jacomy 1990]. Sa machine à percer marque en effet l'ouverture d'une nouvelle période que B. Gille qualifie d'essor du machinisme (7). Vaucanson est en effet représentatif d'une époque transitoire : la fin de la quête du mouvement perpétuel et le début de l'ordre industriel où les machines ont une finalité bien précise.

Si le $\mathrm{XIX}^{\mathrm{e}}$ siècle est marqué par un essoufflement de la construction d'automates, le support matériel des machines s'améliore avec une énergie bon marché ainsi que des matériaux plus résistants qui permettent de concevoir de nouvelles réponses matérielles à des besoins techniques existants. Cette tendance s'accentue au $\mathrm{XX}^{\mathrm{e}}$ siècle avec l'apparition synchronisée de plusieurs corps de savoir qui génèrent d'importantes « interrelations technologiques » [M. Frankel 1955].

\section{Le développement de la robotique}

Après la seconde guerre les créations se stabilisent avec la naissance de la cybernétique et la volonté d'utiliser les principes techniques tels que l'asservissement mécanique, l'électricité et l'électromagnétisme dans des automates fonctionnels. En effet le développement à des fins militaires des mécanismes dits d'asservissement dont les radars, permet la mise au point de nouvelles solutions techniques. Parallèlement à la diffusion de nouveaux principes scientifiques, les recherches américaines s'accélèrent avec notamment les automates cybernétiques de G. Walter reposant sur une interaction avec l'environnement (8).

(4) Au sujet de la Renaissance en tant que période charnière voir B. Jacomy, 1990, pp. 131-187

(5) Les principaux automates ludiques de Léonard de Vinci (1452-1519) sont des lions animés et des oiseaux ainsi que les jeux d'eau qui démontrent une parfaite maîtrise du système de régulation interne. Pour plus de détails, voir A. Doyon et L. Liaigre (1979).

(6) Vers le $\mathrm{XVII}^{\mathrm{e}}$ siècle on accepte que les mouvements peuvent se combiner entre eux sans se géner (Descartes, Roberval et Pascal). On commence à distinguer la force de la vitesse : Descartes ouvre le mouvement, Huygens le complète et Newton donne une notion précise de la force. Pour plus de détails voir R. Blanché (1979).

(7) À ce sujet voir B. Gille et P. Naville, « Le machinisme ", Encyclopédie Universalis, 1979.

(8) Pour plus de détails à propos de la naissance de la cybernétique, voir A. Doyon et L. Liaigre, 1979. 
Dans cette même période plusieurs pays dont la Grande-Bretagne, la Norvège, et les États-Unis conçoivent simultanément les premiers robots donnés ou utilisés dans l'industrie automobile (9). G. Devol cède en effet son prototype créé en 1957, à la firme General Motors pour une utilisation dans le secteur de la fonderie. La société norvégienne Trallfa installe sa création (robot de peinture conçu en 1966) dans la même industrie ; par contre l'ingénieur anglais (1957) ne trouve pas de secteur utilisateur. Si les trois prototypes apparaissent identiques au niveau des développements technologiques, il faut souligner que le robot américain de par sa conception originale sera le " dominant design " pour reprendre le concept de W.J. Abernathy et J.M. Utterback et servira de modèle aux pays européens ainsi qu'au Japon.

Pendant que les États-Unis jouent un rôle pionnier, au niveau de l'élaboration de prototypes, les pays de la CEE ne restent pas inactifs. Leur développement de la robotique dépendra des structures institutionnelles, organisationnelles ainsi que des accords de R\&D entre firmes. En France par exemple, la recherche fondamentale s'est très vite structurée permettant des développements technologiques originaux notamment au niveau de la robotique mobile dans le LAAS de Toulouse, de la robotique en milieu hostile à travers les réalisations du CEA, et de la robotique en milieu agricole à travers les travaux de Larfa. Les centres de recherche appliquée sont restés plus rares (à l'exception du LAM de Montpellier). Les firmes automobiles quant à elles sont très impliquées dans ce processus, en développant leur propre activité robotique (la Régie Renault et la firme Citroën).

En Allemagne par contre les recherches fondamentales dans la robotique étaient quasi inexistantes et se développèrent plus tardivement (10). L'atout résida plus dans d'importants accords de coopération avec les firmes japonaises pour la commercialisation et le développement des produits, une conversion progressive des firmes issues du secteur mécanique et surtout dans d'importants instituts de recherche appliquée (les instituts de recherche Fraunhofer tels que IPA à Stuttgart, IPK à Berlin...) servant de passerelles entre les recherches universitaires et les besoins des firmes (des plus petites PME aux plus gros utilisateurs) [N. Lazaric 1991]. Dès le milieu des années 70 la firme Volkswagen produisait ses propres robots ainsi que la firme Daimler Benz (robots hydrauliques de soudage). Cette dernière a eu une stratégie pionnière en introduisant en 1971 les robots Unimate pour la première fois en Europe.

(9) Le terme de robot fut introduit par l'écrivain de science fiction Karel Capek et désigne le terme de travailleur. À l'heure actuelle cette définition est beaucoup plus précise et selon l'institut américain du robot, « un robot est un opérateur reprogrammable et multi-fonctionnel, conçu pour mouvoir des pièces, des outils ou appareils spécialisés, suivant la séquence de mouvements variable et programmée en vue de l'exécution d'une variété de tâches". Cette définition repose sur la capacité d'opérer automatiquement sans surveillance, donc soit d'avoir une mémoire programmable ou des mécanismes ajustables qui commandent la manipulation. Il n'existe pas de définition internationale, ce qui permet à chaque pays de définir ce qu'il entend par robot et d'avoir une différence d'évaluation et de comptabilité des robots. Ainsi on estime que la diffusion des robots japonais est largement surestimée par les statistiques qui prennent en compte les simples manipulateurs qui n'entrent pas dans la définition de robot en Europe et aux États-Unis.

(10) Actuellement les recherches fondamentales en Allemagne sont beaucoup plus développées à travers notamment le programme de robotique mobile Artes soutenu par les firmes Thyssen, AEG et ESG et qui par de nombreux aspects ressemble au programme anglais Mari (Mobile advanced robotics initiative) regroupant de nombreux industriels sous la houlette de British Aerospace. 
Si les pays européens connaissent chacun un développement différencié, suivant les systèmes nationaux d'innovation - selon l'acception de B. Lundvall -, il faut noter certaines similitudes encore aujourd'hui, au niveau de l'implication des constructeurs du secteur automobile dans cette création (Renault, Volkswagen mais aussi Fiat). Cette participation n'est pas neutre et fait évoluer très sensiblement la trajectoire technologique, en privilégiant certaines applications spécifiques, le soudage notamment [N. Lazaric 1990].

\section{II. - L'ÉLECTRONISATION PROGRESSIVE DES DIFFÉRENTS SOUS-ENSEMBLES TECHNOLOGIQUES}

Le robot à l'heure actuelle est formé d'un ensemble de corps de savoir interdépendants dont le sous-ensemble mécanique, le sous-ensemble de commande, le sous-ensemble de motorisation et le sous-ensemble d'équipement sensoriel. Ces derniers vont profondément évoluer face au développement de l'électronique, modifiant considérablement la conception. Cependant il faut souligner le caractère relativement continu de la diffusion de ce nouveau corps de savoir. On ne peut pas en effet parler de rupture au sens schumpéterien de ce terme. Les rendements croissants d'adoption associés à cette nouvelle technologie sont en effet lents à se déclencher et se localisent plus spécifiquement dans le système de commande et au niveau de la motorisation.

Leur origine est la suivante :

- le processus d'apprentissage et plus spécifiquement le " learning by using " : quand les problèmes de sécurité seront résolus lors de l'utilisation de l'électronique, l'adoption de cette technologie sera croissante.

- La nature de l'information diffusée : à partir du moment où l'on connaîtra mieux les caractéristiques de performances de la technologie électronique, on pourra mieux l'utiliser ce qui produira un rendement croissant d'information.

- L'amélioration du dispositif matériel : le perfectionnement du support matériel permet d'augmenter la performance des circuits intégrés et accroît les « interrelations technologiques" en rendant parfois les autres technologies désuètes.

- Les économies d'échelle : à partir du moment où les composants électroniques seront produits en grande série leur prix baissera, en favorisant leur adoption (11).

L'adoption croissante de l'électronique se heurtera cependant à la dynamique des anciennes technologies et à leur capacité à se régénérer en améliorant leurs performances (N. Rosenberg 1982, D. Sahal 1981). Les technologies telles que l'hydraulique ou la technique relais (électromécanique) se combineront avec les nouvelles et formeront dans une période transitoire des combinaisons symbiotiques pour répondre à la diversité des utilisations. Ces combinaisons sont en effet

(11) Les rendements croissants d'adoption auxquels nous faisons référence sont développés dans B. Arthur (1988), exception faite des « externalités de réseau » développées dans le point suivant. 
l'association durable et réciproquement profitable de plusieurs technologies, impulsées par les contraintes industrielles. De plus le choix de certains utilisateurs, les décisions stratégiques des producteurs, seront cruciales pour la création robotique et la localisation de certaines solutions techniques.

\section{L'évolution de la motorisation}

La motorisation présente tout d'abord différentes alternatives technologiques : électrique, hydraulique ou pneumatique reposant sur des supports matériels différents (avec toutefois des dispositifs matériels communs pour l'actionneur hydraulique et pneumatique). La maîtrise de la motorisation électrique va s'avérer relativement longue. Tant que les problèmes de déflagration ne seront pas résolus pour certaines applications (la peinture notamment), ce procédé sera peu diffusé, même si le système hydraulique comporte d'importantes contraintes (ce dernier est en effet encombrant avec l'utilisation des pompes, clapets, raccords de tuyauteries et des fuites qu'il occasionne).

Ainsi la solution électrique ne s'imposera que graduellement. En effet de 1973 à 1979, l'actionneur électrique est passé de 8 à $17 \%$ dans la population de robots et ceci au détriment de la solution pneumatique reculant de 39 à $29 \%$ et conjointement à une quasi-stabilisation de la solution hydraulique passant de $51 \%$ à $53 \%$ pour la même période (12). Cette diffusion progressive est due à plusieurs raisons. La première est l'amélioration du dispositif matériel hydraulique et sa miniaturisation. La seconde est la variété des besoins des utilisateurs : si la solution électrique est la plus maniable, elle est aussi la plus coûteuse, ce qui va contribuer à freiner son développement dans les PME. D'autre part le développement de combinaison symbiotique permet d'allier les avantages des technologies (plus spécifiquement la précision de l'électrique et la stabilité de l'hydraulique). La solution hydro-électrique va donc représenter à terme une alternative intéressante pour les petits utilisateurs offrant l'avantage de s'associer aux dispositifs matériels existants.

Cependant l'actionneur hydraulique reste encore aujourd'hui en France une application privilégiée pour certains constructeurs d'automobiles. La Régie Renault utilise en effet ce système hydraulique pour les applications de soudage, permettant de réaliser un plus grand nombre de points de soudure avec un nombre identique de robots (en comparaison avec les autres constructeurs). La Régie bénéficie ainsi de cette solution technique spécifique qui lui permet d'avoir un rapport puissance masse très supérieur en comparaison avec la solution électrique. Elle reçoit les fruits d'un important apprentissage le " learning by interacting » selon le concept de B. Lundvall qui engendre des innovations mineures (amélioration du procédé de filtrage et de l'obturation des composants), tout en se comportant comme un " lead user " - selon l'acception de E. von Hippel - lors du développement robotique dans la firme Renault Automation. Si les bénéfices pour la Régie sont importants, ils sont cependant peut-être moindres pour la firme robotique dont

(12) Les données extraites sont tirées de l'enquête réalisée en France par E. Dombre et A. Liegeois auprès d'un échantillon de 132 firmes en 1979 et 108 en 1973.

E. Dombre et A. Liegois, « Analyse des robots industriels. Relation entre structure, performance et fonctions ", LAM, 1979. 
les produits spécifiques rendent difficile la vente de systèmes de soudage pour d'autres utilisateurs privilégiant le tout électrique...

\section{La compétition dans le sous-ensemble de commande}

La compétition entre différents systèmes de commande ne fut pas uniquement basée sur des principes techniques mais aussi sur le concept de la programmation. En effet lors de l'élaboration de la machine outil aux États-Unis deux principes virent le jour, un principe analogique reposant sur une commande par apprentissage où l'opérateur élabore la trajectoire de la machine et un principe numérique dont le procédé plus abstrait ne nécessite pas l'intervention de l'opérateur et implique plus les ingénieurs de production. Les autorités américaines US Air Force, le laboratoire du MIT et la firme General Electric réussirent à détourner l'attention des industriels américains pour imposer le procédé numérique plus sophistiqué, évitant l'erreur humaine et permettant aussi à la direction des firmes de conserver le contrôle de la programmation de la machine [D. Noble 1984, J. Perrin 1988].

Le problème de la programmation se posa avec la même actuité lors du développement des premiers robots. Cependant l'histoire fut quelque peu différente dans la mesure où le principal acteur de la robotique $\mathrm{J}$. Engelberger, président de la firme Unimation décida avec l'accord de G. Devol de développer la technique par apprentissage, moins élégante que la commande numérique mais jugée plus pratique, moins complexe et finalement plus adaptée aux besoins des utilisateurs. Cette décision au début des années 60 ne fut pas isolée puisque AMF dans la même période lors du développement de son robot Versatran, utilisa ce même procédé. Ainsi pendant de nombreuses années et encore aujourd'hui, la commande par apprentissage va subsister dans les firmes utilisatrices, avec une évolution graduelle vers l'intégration de l'électronique qui modifie le principe de programmation par l'opérateur.

Cette progression de l'électronique se fera dans deux directions précises :

- au niveau de la commande relais (commande logique au dispositif technique ancien remontant aux automates de Vaucanson et reposant sur une articulation aller retour). Les manipulateurs " tout ou rien » ou robot pick and place servant aux fonctions de manutention, de palettisation et de déchargement vont progressivement incorporer des processeurs spécialisés, donnant naissance à une nouvelle combinaison symbiotique, l'automate programmable.

- Au niveau de la programmation par apprentissage dont l'asservissement utilisera de plus en plus l'aide électronique pour la commande de la trajectoire, tout en s'appuyant sur l'aide de l'opérateur. Les applications sont dans le domaine du soudage, de la peinture, découpe et assemblage. Ce procédé est de plus en plus réalisé en " hors ligne » permettant de réduire le temps d'élaboration de la programmation pendant la montée en cadence.

L'électronisation du robot industriel est notamment due :

- à une meilleure évaluation des caractéristiques de performances produisant des rendements croissants d'adoption ; 
- à d'importantes interrelations technologiques et l'amélioration des technologies affluentes (évolution des circuits entrées — sorties, mémoires d'interface...) ;

- et surtout d'importantes économies d'échelle pour les microprocesseurs faisant très sensiblement diminuer leur coût. Comme le souligne J. Egelberger, lors de la construction des premiers robots les coûts de l'électronique s'élevaient à $75 \%$ du coût total : le reste étant lié aux coûts de la partie hydro-mécanique. Or au milieu des années 80 , la baisse des prix des composants électroniques a inversé cette proportion!

Actuellement l'emploi de la commande électronique et du microprocesseur est de plus en plus un processus irréversible touchant plus de $80 \%$ de la population des robots [P. Coiffet 1986]. D'une part ses caractéristiques de performance se sont accrues (il peut maintenant effectuer plusieurs opérations simultanément alors qu'auparavant il ne pouvait réaliser qu'une opération à la fois même si le délai d'exécution était extrêmement rapide). De l'autre cette technologie est de plus en plus reliée à d'autres multi-processeurs ou systèmes de données à différents niveaux de l'atelier générant une interaction pendant la programmation avec la possibilité d'un contrôle du process en temps réel. Si les interrelations technologiques sont importantes, la progression de l'électronique et surtout de l'opto-électronique a cependant des limites liées aux capacités d'apprentissage des firmes utilisatrices, que nous allons examiner plus attentivement.

\section{III. - APPORT DE TECHNOLOGIES AFFLUENTES ET IMPLICATION DES ACTEURS}

Dans les années 90 le robot s'insère de mieux en mieux dans le tissu industriel au fur et à mesure qu'un certain nombre de problèmes techniques sont résolus : la motorisation électrique n'est plus dangereuse, les performances du microprocesseur sont mieux connues et mieux évaluées. La maîtrise de ces solutions permet la constitution d'un savoir-faire tant du côté des utilisateurs que des concepteurs et constitue un "learning via diffusion " indispensable pour l'obtention d'une information satisfaisante sur une technologie complexe [D. Sahal 1981].

Pendant que la conception du robot se stabilise avec la maîtrise des innovations, de nouveaux « corps de savoir » se constituent autour de l'opto-électronique. Ces technologies sont dans la continuité de la trajectoire précédente de l'électronisation de la firme. Alors que les performances technologiques pourraient être accrues (grâce à une meilleure interactivité le long du process et une plus grande flexibilité), le développement de la robotique est freiné par le manque de coordination des acteurs autour de la normalisation du stockage des données et surtout par une remise en cause des seuls principes technologiques comme garants d'une automatisation réussie (13)...

(13) La flexibilité que nous évoquons ici est technologique, elle entraîne bien sûr une " flexibilité dynamique » et donc une meilleure réactivité par rapport à la demande (P. Llerena et P. Cohendet 1989). 


\section{L'opto-électronisation des instruments de mesure et les limites de la sophistication technologique}

Ces nouveautés permettent une évolution sensible du sous-ensemble de l'équipement sensoriel. Ce sont en effet des capteurs favorisant l'échange d'informations avec le sous-ensemble de commande et conditionnant ainsi l'évolution de ce dernier. Il existe deux sortes de matériels :

- les capteurs dits internes apportant des informations sur l'exécution du mouvement ;

- et les capteurs externes renseignant sur l'environnement du robot.

Les premiers se sont diffusés progressivement dans le robot et incorporent de plus en plus d'électronique ce qui les rend plus fiables et précis (14). Cependant au début des années 80 ces équipements étaient peu présents car trop sophistiqués et peu adaptés à l'environnement industriel. Comme le souligne A. Perez « les capteurs de mesure sont encore trop chers et trop fragiles pour s'intégrer dans un environnement de vibrations et de poussières. Quand on vise les sommets de la performance, les limites sont le temps réel c'est-à-dire l'informatique. Paradoxalement, cette science est à la fois le frein et le moteur de la robotique " (15). Si ces solutions techniques se développent pas à pas, l'application de l'" intelligence " artificielle va faire évoluer cette technologie. Il ne s'agit pas à proprement parler d'une " intelligence " mais d'une plus grande interactivité améliorant les informations transmises au microprocesseur (cette interactivité s'obtient à travers une communication bidirectionnelle et se réalise grâce à l'intégration d'un microcontrôleur). L'échange de données favorise l'interrogation du système de commande en temps réel et donne la possibilité de modifier la commande du robot pendant la production. Ces capteurs dits " intelligents » utilisent des transmissions numériques dans un grand nombre de cas contrairement aux capteurs « classiques " qui reposent sur le principe analogique (16).

À l'heure actuelle leur diffusion se trouve limitée car l'environnement technologique nécessite d'être reconfiguré au niveau des transmissions de données, ce qui ne peut être réalisé uniquement que par des utilisateurs importants tels que les firmes du secteur automobile. Cette restructuration s'inscrit dans un processus d'apprentissage technico-organisationnel beaucoup plus large qui comprend la modification du process, des méthodes de travail et du produit (qui doit être repensé pour faciliter son automatisation).

(14) Les capteurs d'information (internes) ne sont cependant pas un groupe homogène. Il existe en effet les capteurs de vitesse et les capteurs de position qui mesurent le déplacement du robot soit par rotation ou translation.

(15) A. Perez, " Les capteurs maillon faible ", dans Industries et Techniques $n^{\circ} 441$, déc. 1980. Pour en savoir plus voir aussi F. Lebel, « À capteurs modestes, automatismes médiocres ", dans Industries et Techniques $\mathrm{n}^{\circ} 441$, déc. 1980.

(16) La différence principale entre un capteur numérique et un capteur analogique est que le premier comporte une boucle de rétroaction qui constitue un " prétraitement " de l'information avant l'envoi au système de commande, alors que le second envoie directement cette information. 
Les seconds capteurs touchés par l'introduction de l'" intelligence » artificielle sont les capteurs de vision qui prélèvent une information à distance pour la transmettre indirectement au système de commande (17). Les plus courants sont constitués d'un système de caméra associé parfois à un système de laser. Ces capteurs sont " intelligents " car ils génèrent une meilleure " compréhension " et " perception » de l'environnement. Leur diffusion reste graduelle et au milieu des années 80 la vision associée à la robotique ne représente que $10 \%$ de l'ensemble des systèmes de vision (18). Ces technologies, encore aujourd'hui relativement simples, utilisent des capteurs de vision de deux dimensions (en 1989 on estime que 80 à $90 \%$ du marché européen est constitué de vision plane à deux dimensions) (19). En effet les systèmes de vision tridimentionnelle en sont à leur " balbutiement " (pour la dépose d'un pare brise le système de vision associé à la robotique ne peut distinguer que la carrosserie rouge avec son plan laser et ne fonctionne pas lorsque la couleur est blanche ou noire!).

Les performances de la vision peuvent être un élément clé pour le développement de la robotique en accroissant de façon substantielle la flexibilité. Ces systèmes ont la capacité de gérer sur la même ligne d'assemblage des fabrications à la fois spécifiques (pose d'un volant à droite) et standards pour permettre un assemblage en temps réel (20). Les capteurs " intelligents " internes ont les mêmes possibilités notamment au niveau de l'interaction sur l'opération en cours et évitent par exemple la production de 30 voitures avec le même défaut. Cependant leur mise en place implique en parallèle une gestion du stockage des données qui modifie en profondeur la firme et génère la mise en place de réseaux locaux industriels facilitant les liaisons avec un bus de données indispensable pour l'interconnexion de l'ensemble de ce système.

La très faible diffusion des systèmes de vision s'explique d'une part par le coût matériel et la sophistication technologique générée ne pouvant être supportée que par des gros utilisateurs. On assiste d'autre part dans les firmes du secteur automobile (Renault, Peugeot mais aussi Volkswagen) à une simplification des procédés existants et un retour aux solutions techniques évitant la complexité technologique et la multiplication des pannes lors de la montée en cadence. Les systèmes de vision dans le site de Douai de la Régie Renault par exemple furent très mal adaptés aux conditions industrielles, très différentes des applications en laboratoire et ne furent pas reconduits lors de la robotisation de la Clio à Flins. BMW et Daimler Benz après avoir testé ces systèmes dans leur laboratoire, ont refusé d'implanter cette technologie jugée encore trop fragile au niveau de la fiabilité des capteurs.

(17) Il existe plusieurs types de capteurs de vision : les capteurs infra-rouge (où l'émetteur envoie un faisceau infra-rouge sur l'objet à travers une lentille), les capteurs ultra sonores et les plus courants que nous évoquons ici : les systèmes de caméra.

(18) Les données extraites sont pour l'année 1986. Source : E. Catar, "Quel sens pour les robots ?", Électronique industrielle, $\mathrm{n}^{\circ} 126,1987$.

(19) Données extraites d'Industries et Techniques du 7 décembre 1991.

(20) Certaines firmes du secteur automobile utilisent des systèmes de vision pour le suivi de joint et la dépose d'un pare brise ; Volkswagen notamment pour améliorer la fiabilité du montage des roues et la flexibilité de son système d'assemblage (pose du volant). 
De plus il existe dans les groupes français notamment, une remise en cause de la culture techno-scientiste - selon l'acception de M. Freyssenet - qui tend à ne plus légitimer le seul rôle de la technologie. Cette dernière ne s'impose plus comme une donnée dans la mesure où elle s'appuie sur les compétences humaines et connaissances des opérateurs, mais devient une véritable création collective.

Si l'automatisation n'est pas remise en cause dans le soudage notamment, celle du montage est plus contestée. Dans le groupe PSA, par exemple lors du lancement de la XM à Rennes en 1989, le montage final était robotisé pour le tableau de bord, le pare brise et les roues. Deux années plus tard, la robotisation du montage tend à diminuer pour la $\mathrm{ZX}$ à Aulnay au niveau du montage des roues et est en forte régression pour le nouveau modèle 106 à Mulhouse au niveau des trois applications. Cette « automatisation raisonnable » s'appuie sur un renouvellement socio-technique à travers le plan Mercure chez Citroën, le plan Mides de Renault et le programme Isoar de Peugeot, visant à recomposer l'identité et l'implication des acteurs tout en renouvelant les routines existantes (communication en cas de panne, dépannage du robot...).

\section{Incertitude et coordination des acteurs dans les projets FIP et Profibus}

L'intégration de systèmes opto-électroniques nécessite l'adoption de réseaux locaux - tels que MAP - , pas toujours adaptés aux liaisons d'équipement dans l'atelier. Si l'apparition d'un réseau complémentaire plus en aval permettrait de résoudre ce problème, les utilisateurs européens et instances de normalisation se livrent à une compétition d'une grande âpreté entre deux réseaux, aux conceptions et implications des acteurs radicalement opposées. Le réseau local pour la robotique permet notamment de remplacer les liaisons point par point des équipements au système de commande par une liaison numérique facilitant leur autonomie pour la reprogrammation sans modification des instruments de mesure. Le principal intérêt pour les utilisateurs outre cette flexibilité technologique locale, réside dans la possibilité de connecter différents équipements n'appartenant pas tous au même fournisseur. Ceci permet de choisir les équipements plus librement, de ne pas renouveler les achats en fonction du matériel déjà existant et donc de minimiser les « switching costs » [S.M. Greenstein 1991].

Cependant la compétition entre le projet FIP, norme française soutenue et développée par un groupement d'utilisateurs, et le projet Profibus d'origine allemande émis par le producteur Siemens principalement, est rude et laisse les utilisateurs perplexes. Outre la différenciation technologique pour l'accès au réseau, le principal enjeu réside autour du secteur utilisateur. Le projet français soutenu par EDF et les firmes italiennes du même secteur a une image de réseau local pour process continu alors que le projet allemand soutenu par les firmes automobiles tente d'imposer une image spécifique pour le secteur manufacturier (21). En fait les projets de conceptions technologiques différentes sont relativement similaires dans leur utilisation, mais la compétition particulièrement rude tente d'opposer les pro-

(21) Nous ne développerons pas plus précisément le cas de cette compétition. À noter toutefois que Profibus est soutenu par l'ensemble des constructeurs allemands dont Siemens, alors que la norme française sensiblement très proche de la précédente bénéficie du soutien des utilisateurs dont Cegelec. 
jets avec des stratégies de "pré-annoncements " autour de l'avancement des produits commercialisés, des effets de démonstration et d'implication d'acteurs stratégiques notamment les firmes d'instrumentation (ralliées au projet allemand). $\mathrm{Si}$ les petits et moyens utilisateurs sont dans une position attentiste au niveau de l'adoption, d'autres tentent de créer des évènements par leur implication et leur soutien à des projets qu'ils jugent adaptés à leurs besoins spécifiques.

Ainsi suivant la taille des utilisateurs et leur pouvoir, les firmes ont une capacité à ne pas subir les évènements exogènes mais comme le constatent $\mathrm{M}$. Callon et $\mathrm{V}$. Mangematin à créer des évènements bénéfiques pour leur développement technologique. Dans ce cas précis les évènements deviennent endogènes au processus de compétition et non plus exogènes comme le laisse supposer le modèle de B. Arthur. Les premiers utilisateurs qui doivent supporter les " coûts transitoires d'incompatibilité " ont donc un rôle stratégique et peuvent déclencher des mécanismes d'auto-renforcement surtout s'ils sont importants provoquant d'importantes " externalités de réseau » dans la mesure où ils réduisent les résistances d'adoption et où ils augmentent de par leur choix l'utilité du réseau [M. Katz et C. Shapiro 1985].

L'attentisme des petits utilisateurs se trouve conforté par un certain mimétisme des agents (en période d'incertitude les firmes ont tendance à se conformer aux routines organisationnelles présentes plutôt que d'adopter une technologie non stabilisée). Comme le soulignent S. Metcalfe et G. Dosi : « en un sens, les comportements routiniers, bien que marqués par l'histoire et le contexte de leur apparition, sont des tentatives pour " stabiliser » un environnement non stationnaire... [et]... gouvernent une grande partie des interactions entre agents, fixent les limites de " ce qui est possible de faire ", guident la formation des anticipations " (22). En fait le développement technologique est souvent contingent de l'interdépendance du choix des firmes réalisé de façon séquentielle. À partir du moment où la technologie est choisie les mécanismes de rétroaction positive et de rendements croissants d'adoption la localisent vers une trajectoire de plus en plus irréversible. Ceci souligne l'importance de la coordination des investissements en période incertaine et de la convergence des choix des firmes qui dépendra de la nature de l'information disponible et de la stratégie des acteurs au moment de l'adoption.

\section{CONCLUSION}

Le développement de la robotique, dont les fondements techniques sont anciens, a été particulièrement bouleversé par l'avènement de l'électronique. Cependant cette trajectoire s'est mise en place progressivement avec une variété de solutions techniques dues à une association d'anciennes et de nouvelles technologies, une cristallisation constituant une étape transitoire vers une adoption croissante. Ainsi l'hydro-électrique dans la motorisation et l'automate programmable sont des exemples de combinaisons symbiotiques freinant la linéarité des rendements croissants d'adoption. De plus la trajectoire vers une électronisation accrue et une sophistication technologique importante, s'est trouvée confrontée à d'importants problè-

(22) G. Dosi et S. Metcalfe, 1991, p. 53. 
mes organisationnels bouleversant les structures de la firme dans la mesure où l'informatisation modifie la transmission des données et nécessite de reconcevoir le flux d'information et donc les routines existantes. La création robotique pour être adaptée à la firme doit s'appuyer sur une évolution organisationnelle et une implication des ressources humaines, détenant l'information et les connaissances du procédé et surtout sachant l'adapter au contexte local.

Il existe donc une limite à la trajectoire de l'électronisation croissante de la robotique, liée aux difficultés des firmes à implanter des systèmes technologiques complexes, peu adaptés aux contraintes industrielles et se traduisant par une multiplication des pannes remettant en cause leur principe. Parallèlement à cette remise en cause de l'automatisation technologique, émergent d'importantes incertitudes quant à l'aboutissement des projets FIP et Profibus, incertitude qui freine l'adoption des réseaux locaux industriels spécifiques à la robotique et retarde les améliorations technologiques, qui isolées de leur contexte socio-organisationnel auront du mal à prendre racine.

\section{BIBLIOGRAPHIE}

W.J. ABERNATHY et J.-M. UTTERBACK : « A dynamic model of process and product innovation ", Omega, vol. 3, 1975.

M. AMENDOLA et J.-L. GAFFARD : " La dynamique économique de l'innovation », Economica, Paris, 1988.

ANNALES DES MINES : " Pour une automatisation raisonnable de l'industrie », numéro spécial, janv. 1988.

W.B. ARTHUR : "Competing technologies an overview ", dans Technical Change and Economic Theory " Dosi et alii (éds), Londres et New York, 1988.

A. ATKINSON et J. STIGLITZ : « A new view of technical change », The Economic Journal, 1969.

G. ATTINGER : «L'horlogerie ", Encyclopédie Universalis, 1979.

R. BLANCHÉ : « Histoire de la logique », Encyclopédie Universalis, 1979.

R. BOYER : «Les figures de l'irréversibilité en économie », HESS, Paris, 1991.

M. CALLON et V. MANGEMATIN, "Technological competition, strategies of the firms and the choice of the first users : the case of road guidance technologies », Colloque " Management de la technologie ", Paris, mai 1991.

F. COCHET : « Origines, conditions et perspectives de l'automatisation de la manipulation dans la production ", Thèse de $3^{\mathrm{e}}$ cycle, ECT Lyon, 1987.

P. COHENDET et P. LLERENA : « Flexibilité, Information et Décision », Economica, Paris, 1989.

P. COIFFET : « La robotique : principes et application », Hermes, Paris, 1986.

B. CORIAT : "L'atelier et le robot ", Bourgeois, Paris, 1990.

P. COSTABEL : « Histoire de la mécanique », Encyclopédie Universalis, 1979.

P. DAVID : " La moissonneuse et le robot ", dans " Les enjeux du changement technologique » de JJ. SALOMON et G. SCHMÉDER, Economica, 1986. 
P. DAVID : « The dynamo and the computer : an historical perspective on the modern productivity paradox ", The American Economic Review, May 1990.

G. DOSI et J.S. METCALFE : « Approches de l'irréversibilité en théorie économique », dans R. Boyer ibid 1991.

A. DOYON et L. LIAIGRE : " Les automates », Encyclopédie Universalis, 1979.

D. FORAY : " Les modèles de compétition technologique : une revue de la littérature ", Revue d'Économie industrielle $\mathrm{n}^{\circ} 48,1989$.

D. FORAY : « Dynamique économique et nouvelles exigences de l'investigation historique : "learning to love multiple equilibria" ", Revue économique, n² 2 , mars 1991.

M. FRANKEL : " Obsolescence and technological change in a maturing economy », dans W.B. Arthur 1988.

M. FREYSSENET : « Les formes sociales d'automatisation », Cahiers du GIP, janv. 1990.

B. GILLE : «L'histoire de la technique », La Pléiade, Gallimard, 1978.

S.M. GREENSTEIN : « Lock in and the costs of switching mainframe computers vendors : what do buyers see ?, Working Paper, Université de Stanford, avril 1991.

A. D'IRIBARNE : « La gestion de l'organisation et des ressources humaines comme facteur stratégique de la production et de la diffusion d'innovation ", REI, $\mathrm{n}^{\circ} 51$, premier trimestre 1990.

M. HANADA et N. LAZARIC : " Analyse comparative du contexte institutionnel et organisationnel de la diffusion de la productique dans l'industrie : les cas du Japon et de l'Allemagne ", Étude réalisée pour la SGDN, juillet 1991.

B. JACOMY : «Une histoire des techniques ", Seuil, Paris, 1990.

M. KATZ et C. SHAPIRO : " Network externalities, competition and compatibility », dans American Economic Review, vol. 75, 1985.

N. LAZARIC : «Interactions between user and producer in robotic sector ", Contribution to Fast programm, sept. 1990.

B.A. LUNDVALL : «Innovation and interactive process : from user-producer to national systems of innovation ", dans Dosi et alii eds., Technology and economic theory, Frances Pinter, London 1988.

D.F. NOBLE, "Forces of production - a social history of industrial automation ", Oxford University Press, 1984.

N. ROSENBERG : « Perspectives on technology », Cambridge University Press, 1976.

N. ROSENBERG ; «Inside the black box », Cambridge University Press, 1982.

D. SAHAL : « Patterns of technological innovation », Addison Wesley, 1981.

E. von HIPPEL : « Lead user analyses : a source of novel product concepts », Management Science, $32,1986$.

E. ZUSCOVITCH : «Une approche méso-économique du progrès technique », thèse d'État, Strasbourg, 1984. 